\title{
A MOVING BOUNDARY DIFFUSION MODEL FOR PIN DIODES
}

By:

H. Zhang

J.A. Pappas

10th EML Symposium, April 25-28, 2000, San Francisco, CA

IEEE Transactions on Magnetics, vol. 37 no. 1, January 2001, pp. 406-410

PR - 272

Center for Electromechanics

The University of Texas at Austin

PRC, Mail Code R7000

Austin, TX 78712

(512) 471-4496

April 13, 2000 


\title{
A Moving Boundary Diffusion Model For PIN Diodes
}

\author{
Hanzhong Zhang and John A. Pappas \\ The University of Texas Center for Electromechanics
}

\begin{abstract}
A large number of diode models exist that simulate the reverse recovery process. Many models assume an abrupt change of current during reverse recovery. Some models were verified by calculating the diode's response after the application of a step forcing voltage. Only a few models described in the literature compare simulation results with experimental data. The abrupt change in current calculated by most diode models will ensure large di/dt, which in turn will result in the calculation of an excessive voltage spike. The diode model described in this paper is aimed at the application of high power rectification where the exciting voltage is more likely to be sinusoidal rather than a step change. The formulation is particularly useful in modeling very high power systems such as electromagnetic launch systems where calculation speed and accuracy of results are held at a premium. The model is formulated based on a p+in+ type diode. In addition, by considering the fact that the width of the intrinsic bulk region reduces significantly during the reverse biased condition, the model will more accurately calculate reverse recovery current and voltage. Finally, the model is verified by comparing simulation results to experimental data.
\end{abstract}

\section{INTRODUCTION}

$\mathrm{T}$ HE diode is a basic element in modern electronic systems; however, diodes are difficult to model quantitatively. Part of the reason for this difficulty is that diode conduction is best described by nonlinear distributedparameter differential equations that defy the widely used system simulation tools, such as SPICE. The diode model in SPICE assumes an abrupt change of reverse recovery current. This assumption is unlikely to represent most circuit operating conditions, particularly conditions in high power circuits.

Lauritzen and Ma [1] used a modified charge storage model, but the model does not reflect the fact that the intrinsic region width is a function of applied voltage and does not work well in the general LRC circuit. Furthermore, the model lacked verification by experiment. Chua and Chang [2] solved the one-dimensional fixed domain carrier diffusion equation and then approximated it with finite lumped parameters. But again the model does not work very well during the reverse recovery period due to the fact that they ignored the change of the intrinsic bulk region. However, in most cases the lack of

Manuscript received December 21, 1999. The work presented here was funded by the US Army under contract DAAA21-92-C 0105. accuracy during the reverse recovery period is hidden by the inclusion of snubber circuits.

In this paper, the diode model includes the fact that the width of the intrinsic bulk region is a function of applied voltage. The complete diode model includes a combination of a nonlinear carrier diffusion model and a nonlinear junction capacitor model. Assumptions made in formulating the diode model include:

- The diode is $\mathrm{p}+\mathrm{in}+\mathrm{type}$, where the intrinsic bulk region and the charge diffusion length are compatible. Therefore the asymptotic approach used by Chua and Chang [2] does not apply. However, the techniques in commonly used PN junction diodes can be used in this case with a proper factor, as shown by Hall [3].

- The charge carrier lifetime is constant. This is an important assumption in that it allows us to use the Laplace technique to solve the problem.

- The material is either completely depleted of mobile carriers (depleted region) or is completely neutral (bulk region). This is known as the "abrupt space-charge approximation."

- The heavily doped $\mathrm{p}+$ and $\mathrm{n}+$ region has a large number of free carriers. In fact, these two regions are so heavily doped that they behave more like metals than semiconductors.

The level of injected carriers into the intrinsic bulk region is much higher than the level of electron/hole carriers in the equilibrium. This is called high-level injection [4] and is typical for most power diodes. Under this assumption, the electron carrier concentration equals the hole carrier concentration everywhere in the intrinsic bulk region and the electric field is zero. The zero electric field in the bulk region greatly simplifies the analytical work and is a good assumption, as shown by Kurata [5]. In the absence of electric field across the intrinsic bulk region, all the impressed voltage will appear across the depleted regions.

The schematic diode structure which incorporates these assumptions is shown in Fig. 1. Since the two heavily doped regions at the two ends behave more like metals than semiconductors, the carrier distribution in the intrinsic bulk region determines the mode of the diode. For simplicity, equal hole and electron mobilities are assumed, which makes the charge distributions symmetric; therefore, only half of the intrinsic region is needed for analysis. 


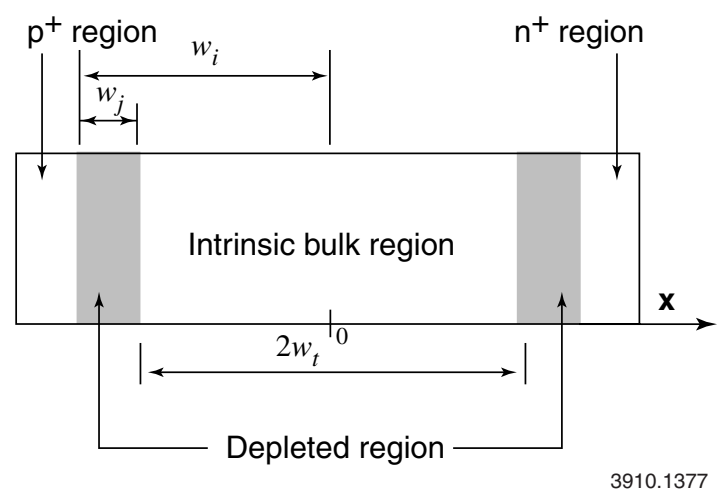

Fig. 1. Schematic drawing of a diode's regions.

The width of the intrinsic bulk region is a function of the applied voltage, as indicated by Neudeck [6] and Streetman [7]. The voltage dependent width eventually makes the charge diffusion model nonlinear, which has significant impact during the reverse recovery period.

Chua and Chang set up their coordinate system at the edge of the bulk region. Since the edge is not fixed in space, this coordinate system is not an inertial system and it is difficult to include the effects of the moving coordinates into the model.

This difficulty could be avoided if we set up the coordinate system at the middle of the intrinsic region as shown in Fig.

1 . We will see that the effects of a moving edge could be easily accounted for by solving nonlinear state space equations, and as a result, the simulation results trace more closely to measurements.

Chua and Chang, however, showed a promising approach to model the distributed system by solving the partial differential equations and approximating the result with finite parameters. A complete diode model can be formulated from parallel-connected systems shown in Fig. 2 (a voltage dependent junction capacitance and a nonlinear charge diffusion model).

\section{JUNCTION MODEL}

There is a junction capacitance associated with the depletion region due to the space charge in the region. The junction capacitance is best described by

$$
C_{j}=-\frac{d Q_{j}}{d v_{j}}
$$

where $Q_{j}$ is the positive space charge and $v_{j}$ is the voltage across the depletion region (junction voltage), which is half of the diode impressed voltage $v$.

Since the capacitance is always positive, (1) shows that if the number of the positive space charges increases (i.e., the depletion region increases), the junction voltage will become more negative (as we expected). By using the fact that the junction voltage is half of the applied voltage, (1) can be rewritten as

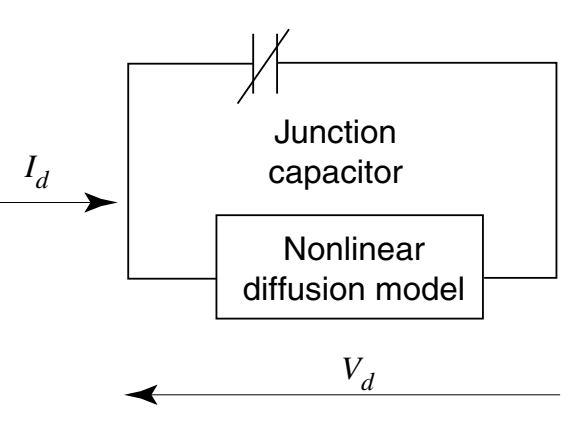

3910.1378

Fig. 2. Diode model topology.

$C_{j}=-\frac{2 d Q_{j} / d t}{d v / d t}$

If we define the current associated with the junction capacitance $C_{j}$, as

$i_{j}=-\frac{d Q_{j}}{d t}$

Equation (2) can be written as:

$\frac{d v}{d t}=\frac{2 i_{j}}{C_{j}}$

The junction capacitance can also expressed in terms of the diode voltage [6]

$$
C_{j}=-\frac{C_{0}}{\left(1-v / V_{0}\right)^{m}}
$$

where $C_{0}$ is the zero-based capacitance of one junction, $V_{0}$ is the built-in voltage, which is assumed to be $1.2 \mathrm{~V}, v$ is the applied voltage, and $m$ varies between $1 / 2$ and $1 / 3$ depending upon the type of junction ( $m=1 / 2$ in this paper). By allowing the factor $m$ to be different from $1 / 2$, we can modify the original abrupt space charge edge assumption. Both $C_{0}$ and $\mathrm{m}$ can be determined experimentally.

\section{DIFFUSION MODEL}

If the origin of the diffusion coordinate is set up in the middle of the diffusion region (Fig. 1), then the carrier diffusion equation can be expressed as

$$
\frac{\partial p}{\partial t}=-\frac{p}{\tau}+D \frac{\partial^{2} p}{\partial x^{2}}
$$

where $\tau$ is the carrier lifetime in seconds and $D$ is the carrier diffusion coefficient $\left(\mathrm{cm}^{2} / \mathrm{s}\right)$.

In order to solve the partial differential equation of (6), further information about the initial condition and the boundary conditions are needed.

There is no loss of generality by assuming a zero initial condition, which is the common starting point in time domain simulations.

The boundary conditions for (6) need a little thought. Since the charge distributions in the intrinsic region are assumed to be symmetric, the slope of the distribution at $x=0$ is always zero. At the edge of the intrinsic region, the 
charge concentration is determined by the junction voltage, as it is in a common pn junction diode. However the width of the bulk region is a function of junction voltage, which in turn is a function of time. Therefore the width of the bulk region is a function of time. In other words, we are dealing with a moving boundary diffusion problem, i.e. a Stefan problem. The Stefan problem is actually a nonlinear partial diffusion equation due to the moving boundary, even though the governing equation is still given by (6).

Therefore the boundary conditions for (6) can be written as:

$$
\begin{aligned}
& \left.\frac{\partial p}{\partial x}\right|_{x=0}=0 \\
& p\left(w_{t}, t\right)=p_{n}\left(e^{v_{j} / V_{T}}-1\right)
\end{aligned}
$$

where

- $p_{n}$ is the equilibrium carrier concentration

- $V_{T}$ is the thermal voltage $(0.0259 \mathrm{~V}$ at room temperature $)$

- $w_{t}$ is the width of the bulk region

Here the subscript $t$ is used to emphasize the fact that the width is a function of time. The relation between the carrier lifetime $\tau$, the charge diffusion coefficients, and the charge diffusion length is: [7]

$$
L^{2}=D \tau
$$

By applying the Laplace transformation on both sides of (6), it can be shown that the charge distribution in the frequency domain is

$$
p\left(x, s, w_{t}\right)=p_{n} R(s) \frac{\cosh (\sqrt{1+\tau s} x / L)}{\cosh \left(\sqrt{1+\tau s} w_{t} / L\right)}
$$

where $R(s)$ is the Laplace transformation of $\left(e^{v_{j} / V_{T}}-1\right)$ or $\left(e^{v / 2 V_{T}}-1\right)$ due to the assumption that the junction voltage $v_{j}$ is the half of the impressed voltage $v . w_{t}$ in (10) can be viewed as a parameter rather than as a space variable.

The hyperbolic function in (10) can be expanded in power series as:

$$
\cosh \left(\sqrt{1+\tau S} \frac{w_{t}}{L}\right)=\sum_{k=0}^{\infty} \frac{(1+\tau S)^{k} w_{t}^{2 k}}{(2 k) ! L^{2 k}} .
$$

Equation (11) shows that $k$ needs to be infinite for an accurate representation. However since the bulk region $w_{t}$ is compatible to the diffusion length $L$, only the first few elements are needed.

The diffusion current that is proportional to the charge distribution slope at the edge of the intrinsic region [7]

$$
i_{\text {dif }}(t)=\left.\frac{A q D}{L} \frac{\partial p(x, t)}{\partial x}\right|_{x=w_{t}} .
$$

This relation also holds true in the frequency domain.

From (10), (11) and (12), we have

$$
i_{\text {dif }}\left(s, w_{t}\right)=I_{0} R(s) \frac{\sum_{0}^{k} \frac{(1+\tau s)^{k} w_{t}^{2 k+1}}{(2 k+1) ! L^{2 k+1}}}{\sum_{0}^{k} \frac{(1+\tau s)^{k} w_{t}^{2 k}}{(2 k) ! L^{2 k}}}
$$

where

$$
I_{0}=\frac{A q D p_{0}}{L}
$$

is defined as the reverse saturation current for long base diode, which is the total thermal generated free charges within the diffusion distance from the edge during the average charge time. The non-linearity arises from the fact that $w_{t}$ is a function of time.

For a given $w_{t}$, (13) can be easily realized in Matlab ${ }^{\circledR}$ by writing a set of state-space equations. The depletion (junction) region width can be expressed as

$$
w_{j}=\frac{\varepsilon A}{C_{j}}=\frac{C_{0}}{C_{j}} w_{0}
$$

where $w_{0}$ is the zero based junction width and $C_{j}$ is the voltage dependent junction capacitance given in (5). Due to the asymmetrically doped junction, the junction region extends primarily into the less doped intrinsic side. The width of the dynamic intrinsic bulk region can be calculated as

$$
w_{t}=w_{i}-\frac{C_{0}}{C_{j}} w_{0}
$$

where $w_{i}$ is the physical intrinsic region width as shown in Fig. 1.

\section{DIODE MODEL}

The complete diode model is the combination of the diffusion and junction models depicted in Fig. 2. The input to the model is the current through the diode. The algorithm for the computation is given below.

- With a junction voltage state, the junction capacitance is found from (5).

- The dynamic width of the intrinsic bulk region is calculated from (16).

- The diffusion current can be found by converting (13) to a set of state space equation. Since the dynamic width of the intrinsic bulk region is compatible with the diffusion length, only the first few elements are necessary. The number of elements used depends on the ratio of $w_{t} / L$ in (13). In most normal cases when $w_{t} / L$ is between $2 \sim 3$, using $k=3$ is sufficient.

- The junction current is calculated by taking the difference between the input current though the diode and the diffusion current.

- The change of voltage state is found from (4).

\section{MODEL VERIFICATION}

The derived diode model was verified by comparing the simulation result to a SPICE simulation and with measurements. The diode under test (DUT) is a Powerex RA20. The RA20 is a $3.2 \mathrm{kA}$ PIN diode. The characteristic data for RA20 are given in Table 1. A trace of the diode current during recovery was obtained from Powerex and is shown in Fig. 3. A constant $5 \mathrm{kA}$ current was applied for 500 $\mu s$ and then decreased at the rate of $133 \mathrm{~A} / \mu \mathrm{s}$. The data from that test are given in Table 2. The model was also applied to 
TABLE 1. Characteristics of THE PoWerex RA20 Diode

\begin{tabular}{lcl}
\hline \multicolumn{1}{c}{ Characteristic } & Term & \multicolumn{1}{c}{ Value } \\
\hline Built-in junction capacitance & $C_{0}$ & $78 \mathrm{nF}$ \\
Built-in voltage & $V_{0}$ & $1.2 \mathrm{~V}$ \\
Carrier concentration & $p_{n}$ & $5 \times 10^{13} \mathrm{~cm}^{-3}$ \\
Thermal voltage & $V_{T}$ & $0.0259 \mathrm{~V}$ \\
Reverse saturation current & $I_{s}$ & $0.0684 \mathrm{~A}$ \\
Carrier concentration & $t$ & $13 \mathrm{~s}$ \\
Carrier diffusion coefficient & $D$ & $12.5 \mathrm{~cm}^{2} / \mathrm{s}$ \\
Physical intrinsic width & $w_{j}$ & $0.025 \mathrm{~cm}$ \\
\hline
\end{tabular}

TABle 2. MEAsured AND CALCULATEd RECOVERy DATA FOR RA 20

\begin{tabular}{lcc}
\hline \multicolumn{1}{c}{ Characteristic } & Measured & Calculated \\
\hline Forward current & $5,000 \mathrm{~A}$ & $5,000 \mathrm{~A}$ \\
Rate of current fall & $133 \mathrm{~A} / \mathrm{s}$ & $133 \mathrm{~A} / \mathrm{s}$ \\
Reverse recovery time & $10 \mathrm{~s}$ & $10 \mathrm{~s}$ \\
Peak reverse current & $1,150 \mathrm{~A}$ & $1,000 \mathrm{~A}$ \\
\hline
\end{tabular}

this diode using the same conditions as the test. The calculated trace is shown in Fig. 4 and the recovery data are given in Table 2 . The results show good agreement between measured and calculated values.

The diode model was also tested under more realistic conditions and at higher than rated currents. The circuit used to test the diode is shown in Fig. 5. The results for two test cases are shown. Figs. 6 and 7 show both measured and calculated traces for an initial voltage of $520 \mathrm{~V}$ and resulting peak current of $6 \mathrm{kA}$. The corresponding SPICE calculations are shown in Figs. 8 and 9. The model used for the calculation is the SPICE diode primitive [8]. The diode characteristics were formulated from data sheet values and measurements carefully made in accordance with the SPICE publisher's instructions [9]. Data sheet values were obtained from [10]. Note the differences between the SPICE calculation and the measurement. The recovery is delayed and the recovery current is calculated to be deeper than what was measured. In addition, the current oscillates about zero long after the recovery period. As a result, the SPICE calculated voltage is much higher than the measured value and the diode appears as though it never holds static reverse voltage. This result would lead a designer to include larger or more diodes in a rectifier than are actually required and would also lead to an incorrect equalization circuit design [11]. Therefore, the resulting system would be more massive and voluminous than required. This result, therefore, is unacceptable for lightweight high-power converter designs. Figs. 10 and 11 show the comparison for the case where the initial voltage is $1,077 \mathrm{~V}$ and the peak current is $12 \mathrm{kA}$.

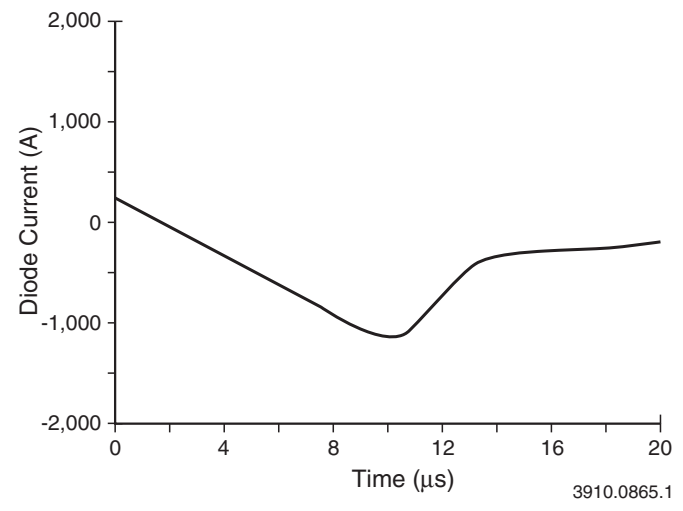

Fig. 3. Powerex RA20 recovery data.

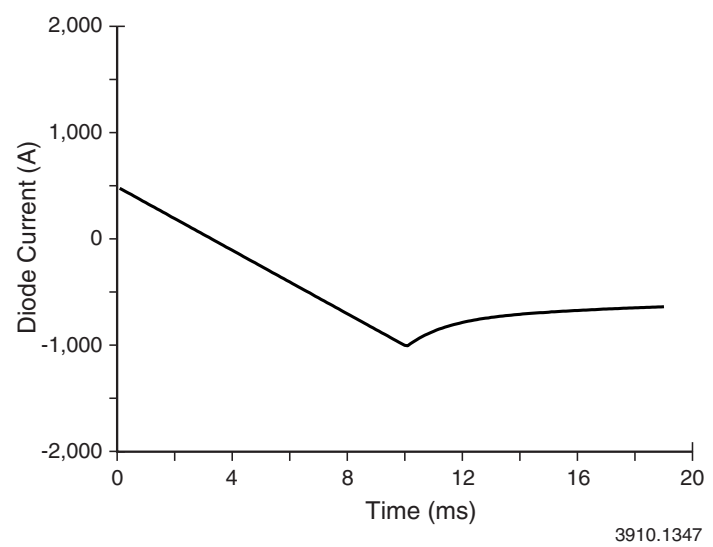

Fig. 4. Simulation of RA20 recovery.

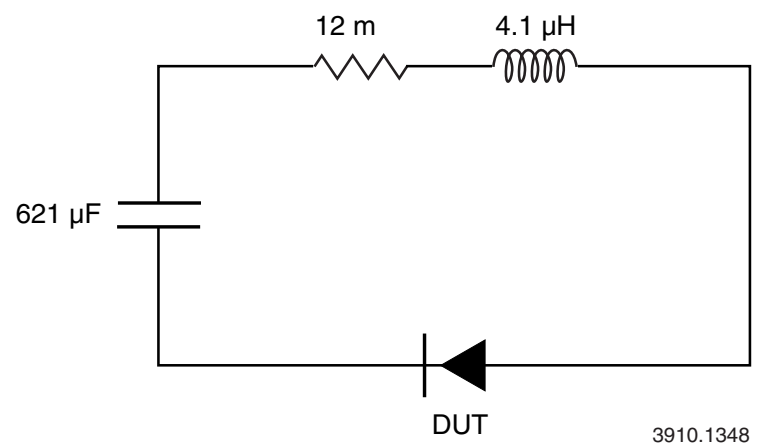

Fig. 5. LRC test circuit. 


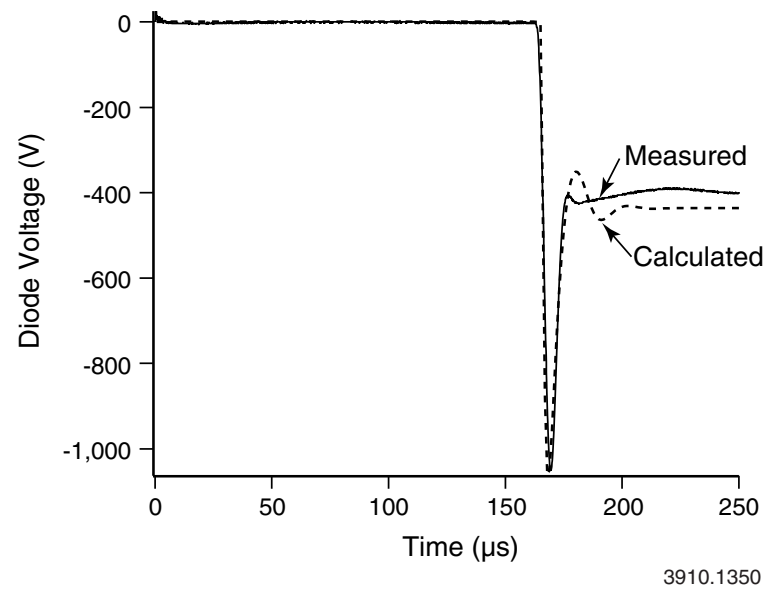

Fig. 6. Comparison of measured and calculated voltage for $V_{0}=520 \mathrm{~V}$.

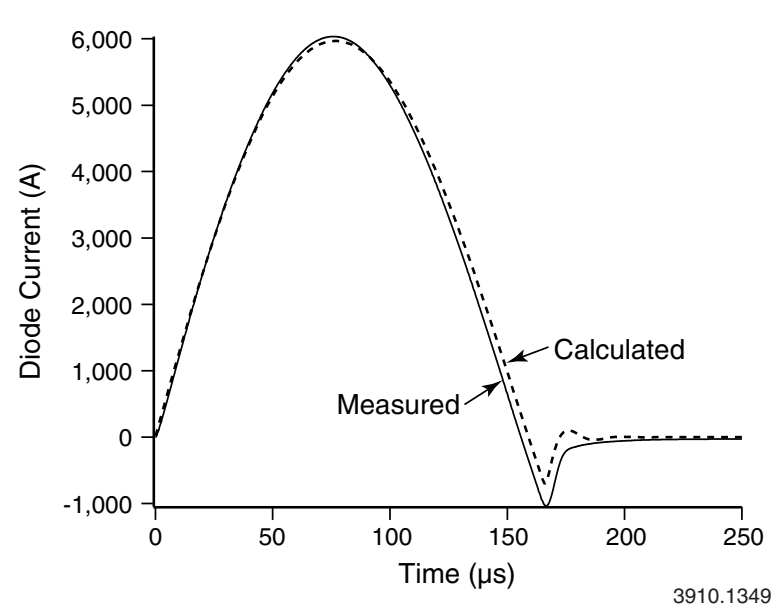

Fig. 7. Comparison of measured and calculated current for $V_{0}=520 \mathrm{~V}$.

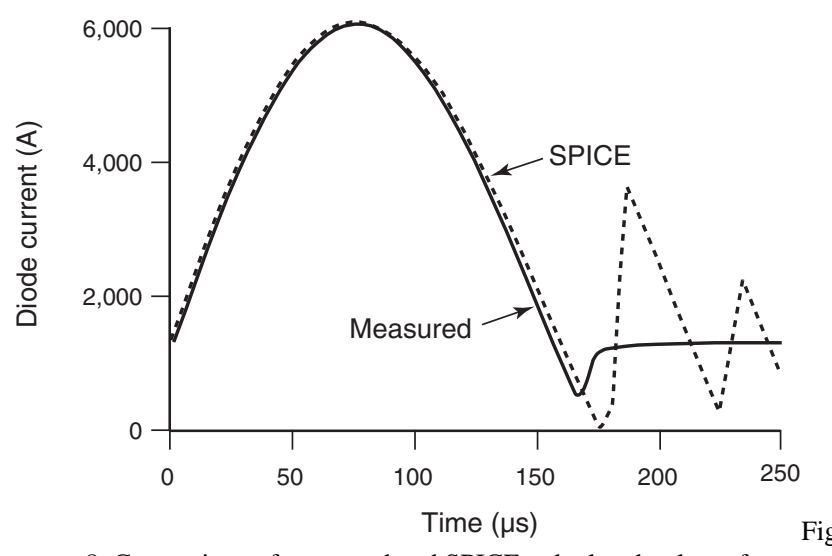

8. Comparison of measured and SPICE-calculated voltage for $V_{0}=520 \mathrm{~V}$.

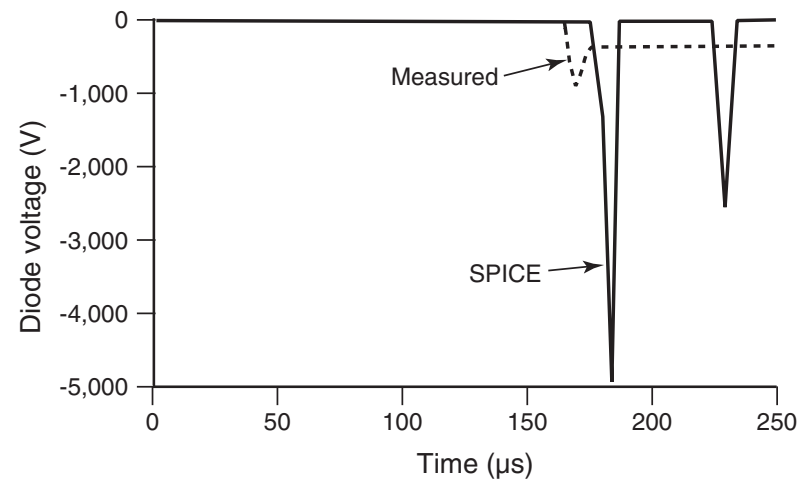

Fig. 9. Comparison of measured and SPICE-calculated current for $V_{0}=520 \mathrm{~V}$.

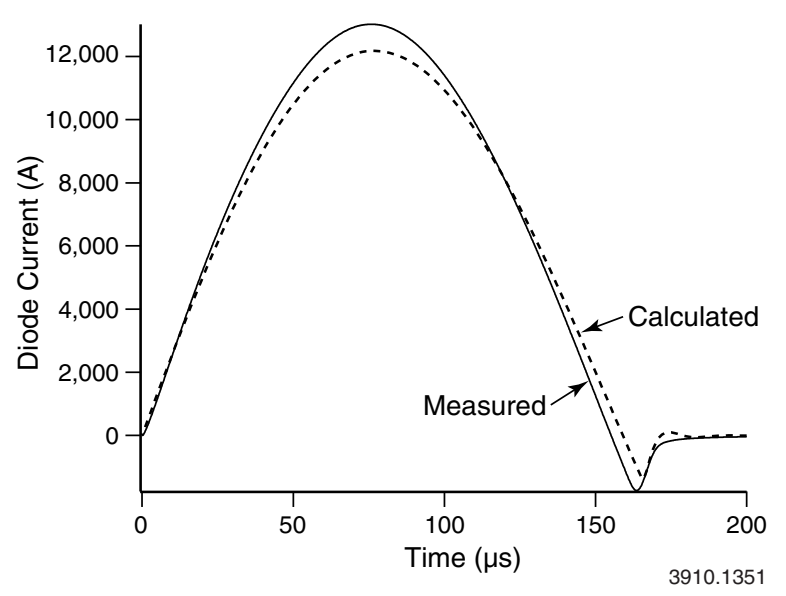

Fig. 10. Comparison of measured and calculated current for $V_{0}=1,1079 \mathrm{~V}$.

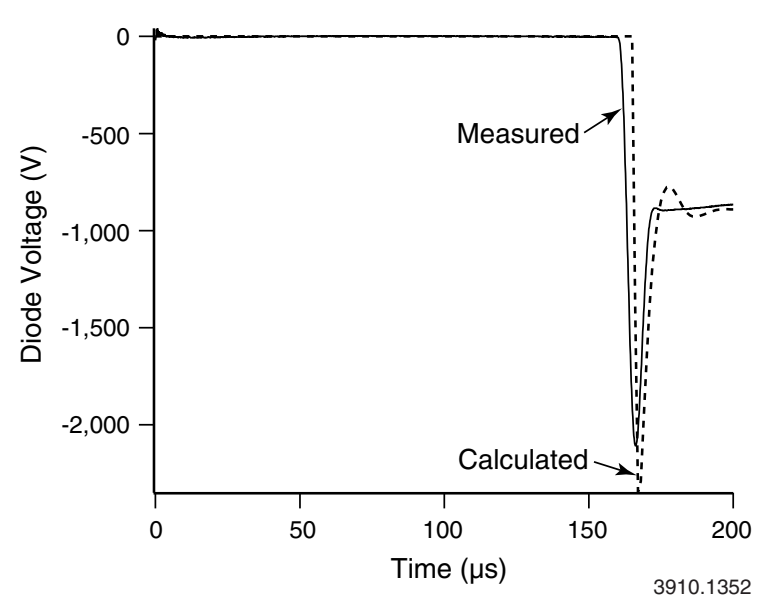

Fig. 11. Comparison of measured and calculated voltage for $V_{0}=1,079 \mathrm{~V}$. 
[11] D.R. Grafham, et al, SCR Manual, $6^{\text {th }}$ ed., Prentice Hall, Englewood Clifs, NJ: 1979.

\section{CONCLUSION}

The experiments clearly show that the model closely predicts diode performance during reverse recovery. The differences between the experimental data and the calculated values lie partly in the approximation of diffusion phenomena and on the assumption that there exist two distinct regions, the bulk region in which there is no electric field and the space charge region in which there are no free carriers. In the real case this is never true. Instead, the boundary is ill defined and the carrier density falls off exponentially into the space charge region. In addition, a mechanical closing switch was included in the test circuit and its effects were not modeled in the simulations. Other deviations are due to the assumptions made in applying the Shockley-Reed recombination theory. The assumption of constant carrier lifetime is not sufficient and the effects of carrier-carrier scattering, Auger recombination, and band-gap narrowing are not considered in the model. However, inclusion of those factors would result in the need to reformulate the diffusion coefficient and carrier lifetime as functions of time. As a result, the Laplace formulation, a major advantage in increasing the speed of the calculation, could no longer be used.

The purpose of the modeling effort was to formulate a turn-off model that was accurate enough to closely design a lightweight power converter without unnecessarily complicating the model and bogging down the power system simulator. The keys to the relative accuracy and reduction in computation time are the facts that the model does not include any active or ideal elements and there are no abrupt changes in model behavior that produce discontinuities in the calculation that slow most numerical integrators.

\section{ACKNOWLEDGMENTS}

The authors gratefully acknowledge the contributions to the device test made by Michael Salinas and Peter Andryshak.

\section{REFERENCES}

[1] P. Lauritzen and C. Ma, " Simple diode model with reverse recovery," IEEE, Trans. Power Electronics,” Vol. 6, No. 2. April 1991

[2] L. Chua, L. and C. Chang, "High-speed nonlinear circuit models for pn junction diodes," International Journal of Circuit Theory and Applications, vol. 16, 157-190, 1988

[3] R.N. Hall, "Power rectifier and transistors," Proc. I.R.E. pg.1512-1518, Nov. 1952

[4] N.H. Fletcher, "The High Current Limit for Semiconductor Junction Devices, Proc. I.R.E., pg.862-872, June 1957

[5] M. Kurata, One-Dimensional Calculation Of Thyristor Forward Voltages and Holding Currents, Solid-State Electronics, 1976, Vol. 19, pp.527-535

[6] G. Neudeck, The PN Junction Diode, 2nd edition Addison-Wesley Publishing Company, 1989

[7] B.G. Streetman, Solid State Electronic Devices, 4th edition, Prentice Hall, 1995

[8] Intusoft, ICAP/4 IsSPICE User's Guide, San Pedro, CA, 1996

[9] Intusoft, ICAP/4 IsSPICE Model Library Listings, San Pedro, CA, 1996

[10] Powerex, Rectifier and Thyristor Applications and Technical Data Book, Youngwood, PA, 1993. 